\title{
SLC30A8 (ZnT8) variations and type 2 diabetes in the Chinese Han population
}

\author{
J. Xu' ${ }^{1}$, J. Wang ${ }^{2}$ and B. Chen ${ }^{1}$ \\ ${ }^{1}$ Endocrinology Department of Southwest Hospital, \\ Third Military Medical University, Chongqing, China \\ ${ }^{2}$ Nutrition Department of Xinqiao Hospital, \\ Third Military Medical University, Chongqing, China \\ Corresponding author: B. Chen \\ E-mail: chenbingxj@yeah.net
}

Genet. Mol. Res. 11 (2): 1592-1598 (2012)

Received March 28, 2011

Accepted February 5, 2012

Published May 24, 2012

DOI http://dx.doi.org/10.4238/2012.May.24.1

\begin{abstract}
In recent genome-wide association studies, variants in the SLC30A8 gene have been found to be associated with risk for type 2 diabetes. We examined a possible association of tag SNPs spanning SLC30A8 and their haplotypes with type 2 diabetes in the Chinese Han population. There were 1508 Chinese Han type 2 diabetes patients and 1500 age- and gender-matched control subjects; all were genotyped for three tagging SNPs (rs2466295, rs4876703, and rs11558471) of the human SLC30A8 gene. The AA genotype of rs 11558471 was found significantly more frequently in type 2 diabetes patients than in controls (46 vs 24\%). The frequency of the A-C-A haplotype was significantly higher in type 2 diabetes patients than in controls $(0.331 v s 0.120)$. The frequency of the A-C-G haplotype was significantly lower in type 2 diabetes patients than in controls $(0.160$ vs 0.365$)$. We conclude that type 2 diabetes is associated with the AA genotype of rs11558471 in the human SLC30A8 gene. The A-C-A haplotype appears to be a risk factor and the A-C-G haplotype may be a protective factor against type 2 diabetes in Chinese Han.
\end{abstract}

Key words: SLC30A8; Type 2 diabetes; Single-nucleotide polymorphism 


\section{INTRODUCTION}

It has been known for decades that type 2 diabetes has a genetic basis (Boutayeb and Boutayeb, 2005), besides the important contribution of environmental factors, including changes in dietary patterns and lifestyle. Over the past decade, serious efforts have been put into the search for type 2 diabetes susceptibility genes, but progress has been slower than anticipated (Das and Elbein, 2006; Owen and McCarthy, 2007). Although several polymorphisms in a few genes including PPARG, KCNJ11, and TCF7L2 have been convincingly replicated in individuals with European ancestry, relatively few studies have been conducted in Chinese. However, recent advances in genome-wide association studies (GWASs) have revived the initial optimism and accelerated the discovery of susceptibility genes for diabetes (Frayling, 2007a,b; Zeggini, 2007). Six high-density ( $>300$ K) GWASs that identify genes affecting risk for type 2 diabetes among Caucasians have been published, and a replicated association was reported with single nucleotide polymorphisms (SNPs) in/near the zinc transporter, member 8 (SLC30A8) gene, one of nine human genes for multispanning transmembrane proteins facilitating $\mathrm{Zn}^{2+}$ efflux from the cell and sequestration into intracellular compartments (Chimienti et al., 2004; Palmiter and Huang, 2004). Although several studies have reported the association of genetic polymorphism of SLC30A8 with type 2 diabetes, much of the research has focused on SNPs rather than haplotypes, which better characterize the common patterns of variation in a population (Crawford and Nickerson, 2005). In the present study, we established haplotypes of the SLC30A8 gene consisting of three SNPs (rs2466295, rs4876703 and rs11558471), and assessed the associations between these haplotypes and type 2 diabetes.

\section{SUBJECTS AND METHODS}

\section{Subjects}

The study sample consisted of 3008 individuals (1508 type 2 diabetes mellitus (T2DM) and 1500 controls) from the Endocrinology Department of Southwest Hospital of the Third Military Medical University and the Nutrition Department of Xinqiao Hospital of the Third Military Medical University. All participants were unrelated Han Chinese, with at least 20 years residence in Chongqing city. All samples were collected following American Diabetes Association guidelines with fasting blood glucose $>120 \mathrm{mg} / \mathrm{dL}$ and/or 2 -h plasma glucose $>200 \mathrm{mg} / \mathrm{dL}$. Case samples were collected after detailed investigation of medical records for symptoms, use of oral hypoglycemic agent and/or insulin, measurement of fasting blood glucose, and other related anthropometric parameters. Subjects diagnosed with type 2 diabetes after the age of 60 or subjects who were started on insulin therapy within one year of diagnosis were excluded from the study. Subjects with type 1 diabetes, family history of type 1 diabetes, maturity onset diabetes of the young, monogenic forms of diabetes, or drug-induced diabetes were also excluded from the study. Samples from patients outside Chongqing and patients migrating from other parts of the country were not included. Case reports of diabetic nephropathy, diabetic retinopathy and fatty liver disease as diagnosed by the physician were also documented during collection. Age-, gender- and ethnicity-matched normoglycemic control subjects were recruited 
in the study by public advertisement and increased awareness by offering screening for diabetic risk factors at specially organized medical camps. All the participants of these medical camps were subjected to a health questionnaire for detailed investigation of their disease status, family history, socio-economic status, food habits, smoking and alcohol status, BMI and blood pressure. Inclusion criteria for the healthy controls were: a) above 40 years of age, b) no family history of diabetes, c) not taking any oral hypoglycemic agent or insulin, d) blood glucose levels $<110 \mathrm{mg} / \mathrm{dL}$.

\section{Genotyping}

There are 1000 SNPs for the human SLC30A8 gene listed in the National Center for Biotechnology Information SNP database (http://www.ncbi.nlm.nih.gov/SNP). Using the Haploview 4.2 software and the HapMap phrase $\beta$ database, we obtained three tagging SNPs (rs2466295, rs4876703 and rs11558471), which represent 14 SNPs (Table 1) crossing $17 \mathrm{~kb}$ of the SLC30A8 gene for Han Chinese using minor allele frequency $\geq 0.10$ and linkage disequilibrium patterns with $r^{2} \geq 0.8$ as a cutoff.

Table 1. Tagging single nucleotide polymorphisms (SNPs) and represented SNPs.

\begin{tabular}{ll}
\hline Tagging SNP & Represented SNPs \\
rs2466295 & rs2466291, rs2466292, rs2464592, rs2466295, rs2466296, rs2466299 \\
rs4876703 & rs6469675, rs10505310, rs10505312, rs13281296, rs4876703 \\
rs11558471 & rs3802177, rs13266634, rs11558471 \\
\hline
\end{tabular}

Genomic DNA was extracted from the peripheral blood leukocytes using a DNA extraction Kit (Beijing Bioteke Co. Ltd.). Quantification of extracted DNA was performed using a NanoDrop ND-1000 spectrophotometer (NanoDrop Technologies, Wilmington, DE, USA). Genotyping was performed using the TaqMan ${ }^{\circledR}$ SNP Genotyping Assay (Applied Biosystems Inc.) as described previously (Livak, 1995; Sano et al., 2005).

\section{Statistical analysis}

Data analysis was performed using the Statistical Package for Social Sciences-SPSS computer software for Windows (version 13.0). Hardy-Weinberg equilibrium was assessed by the chi-square analysis. Measurement data are shown as means $\pm \mathrm{SD}$, and the differences between the T2DM patients and the control subjects were assessed by independent-sample $t$-test. Differences in enumeration data between T2DM patients and control subjects were analyzed using the chi-square test. Differences in distributions of genotypes, alleles, and haplotypes between T2DM patients and control subjects were analyzed using the SHEsis platform (Shi and $\mathrm{He}, 2005$; Li et al., 2009). Pairwise linkage disequilibrium analysis was performed using three SNP pairs. In the haplotype-based case control analysis, haplotypes with a frequency of $<0.03$ were excluded. The frequency distribution of the haplotypes was calculated by performing a permutation test using the bootstrap method. In addition, logistic regression analysis was performed to assess the contribution of the major risk factors. To assess the association of each SNP with T2DM, we used Bonferroni's correction to control for the number of variants tested; this was 3 , so the probability value, $<0.017$, was considered to be significant. 


\section{RESULTS}

Diabetes-related clinical and biochemical parameters were collected for all the case and control samples. Clinical and biochemical features of the study population are summarized in Table 2.

\begin{tabular}{|c|c|c|c|}
\hline & T2DM group $(\mathrm{N}=1508)$ & Controls $(\mathrm{N}=1500)$ & $P$ \\
\hline Age (years) & $53.4 \pm 12.3$ & $53.6 \pm 11.8$ & 0.4585 \\
\hline Systolic blood pressure (mmHg) & $131.2 \pm 11.3$ & $123.6 \pm 11.4$ & $<0.001$ \\
\hline Diastolic blood pressure (mmHg) & $85.7 \pm 9.4$ & $78.3 \pm 9.7$ & $<0.001$ \\
\hline Uric acid (mM) & $344.4 \pm 115.4$ & $347.1 \pm 104.7$ & 0.546 \\
\hline Triglyceride (mM) & $1.70 \pm 0.41$ & $1.69 \pm 0.31$ & 0.407 \\
\hline Total cholesterol (mM) & $4.6 \pm 1.4$ & $4.5 \pm 1.0$ & 0.223 \\
\hline Smoking (N, \%) & $905(60.01)$ & $615(41.0)$ & $<0.001$ \\
\hline Alcohol use (N, \%) & $678(44.96)$ & $660(44.00)$ & 0.734 \\
\hline Pulse (bpm) & $82.5 \pm 14.3$ & $81.1 \pm 13.0$ & 0.129 \\
\hline Body mass index $\left(\mathrm{kg} / \mathrm{m}^{2}\right)$ & $25.1 \pm 2.4$ & $23.8 \pm 2.3$ & $<0.001$ \\
\hline Glucose $(\mathrm{mM})$ & $8.3 \pm 2.7$ & $4.9 \pm 0.6$ & $<0.001$ \\
\hline HbAlc $(\%)$ & $7.97 \pm 0.73$ & - & - \\
\hline Duration (years) & $8.73 \pm 4.57$ & - & - \\
\hline
\end{tabular}

Data are reported as means $\pm \mathrm{SD}$, unless otherwise stated. P values were obtained by comparison of cases and controls; $1 \mathrm{mmHg}=0.133 \mathrm{kPa} ; \mathrm{T} 2 \mathrm{DM}=$ type 2 diabetes mellitus.

The genotype distribution of each SNP was not significantly different from the HardyWeinberg equilibrium values (Table 3 ). The distributions of the genotypes and alleles of these three SNPs are shown in Table 3. The genotype and the allele distributions of rs 11558471 differed significantly between the T2DM patients and the control subjects (both $\mathrm{P}<0.001$ ). The AA genotype and the A allele were more common in T2DM patients than in control subjects. But the genotype and the allele distributions of the other two SNPs were not different between the T2DM patients and the control subjects.

Table 3. Distributions of genotypes and alleles of the SLC30A8 gene in T2DM patients and the control group.

\begin{tabular}{|c|c|c|c|c|c|c|c|c|c|c|c|}
\hline \multirow{2}{*}{$\frac{\text { SNPs }}{\text { rs2466295 }}$} & \multirow[t]{2}{*}{ Group } & \multirow[t]{2}{*}{$\mathrm{N}$} & \multicolumn{3}{|c|}{ Genotypes } & \multirow[t]{2}{*}{$\mathrm{P}$} & \multirow[t]{2}{*}{ HWE } & \multicolumn{2}{|c|}{ Alleles } & \multirow{3}{*}{$\begin{array}{c}\mathrm{OR}(95 \% \mathrm{CI}) \\
1.001(0.87-1.15)\end{array}$} & \multirow{3}{*}{$\begin{array}{c}\mathrm{P} \\
0.986\end{array}$} \\
\hline & & & $\mathrm{A} / \mathrm{A}$ & $\mathrm{A} / \mathrm{G}$ & G/G & & & A & G & & \\
\hline & $\mathrm{T} 2 \mathrm{DM}$ & 1508 & $1071(0.71)$ & $407(0.27)$ & $30(0.02)$ & 0.115 & 0.23 & 0.845 & 0.155 & & \\
\hline & Control & 1500 & $1080(0.72)$ & $375(0.25)$ & $45(0.03)$ & & 0.08 & 0.845 & 0.155 & & \\
\hline \multirow[t]{3}{*}{ rs4876703 } & & & $\mathrm{A} / \mathrm{H}$ & $\mathrm{A} / \mathrm{C}$ & $\mathrm{C} / \mathrm{C}$ & & & A & $\mathrm{C}$ & & \\
\hline & $\mathrm{T} 2 \mathrm{DM}$ & 1508 & $211(0.14)$ & $648(0.43)$ & $649(0.43)$ & 0.07 & 0.05 & 0.355 & 0.645 & $0.895(0.806-0.993)$ & 0.03 \\
\hline & Control & 1500 & $226(0.15)$ & $690(0.46)$ & $584(0.39)$ & & 0.34 & 0.381 & 0.619 & & \\
\hline \multirow[t]{3}{*}{ rs 11558471} & & & $\mathrm{~A} / \mathrm{A}$ & $\mathrm{A} / \mathrm{G}$ & $\mathrm{G} / \mathrm{G}$ & & & A & $\mathrm{G}$ & & \\
\hline & $\mathrm{T} 2 \mathrm{DM}$ & 1508 & $694(0.46)$ & $679(0.45)$ & $135(0.09)$ & $<0.001$ & 0.08 & 0.69 & 0.31 & $2.170(1.96-2.44)$ & $<0.001$ \\
\hline & Control & 1500 & $360(0.24)$ & $781(0.52)$ & $359(0.24)$ & & 0.11 & 0.50 & 0.50 & & \\
\hline
\end{tabular}

$\mathrm{SNP}=$ single nucleotide polymorphism; $\mathrm{HWE}=$ Hardy-Weinberg equilibrium; $\mathrm{OR}=$ odds ratio; $\mathrm{CI}=$ confidence interval; T2DM = type 2 diabetes mellitus.

Table 4 shows patterns of linkage disequilibrium in the SLC30A8 gene, with their $\left|\mathrm{D}^{\prime}\right|$ and $r^{2}$ values. All 3 SNPs are located in 1 haplotype block because all |D'| are beyond 0.5. All three SNPs were available for the performance of a haplotype-based case-control study because all $r^{2}$ values were below 0.5 (Xiang et al., 2009). 
Table 4. Pairwise linkage disequilibrium for the three single nucleotide polymorphisms (SNPs).

\begin{tabular}{|c|c|c|c|c|c|c|c|}
\hline & & \multicolumn{6}{|c|}{$\left|\mathrm{D}^{\prime}\right|$} \\
\hline & & \multicolumn{3}{|c|}{ T2DM patients } & \multicolumn{3}{|c|}{ Control subjects } \\
\hline & & SNP1 & SNP2 & SNP3 & SNP1 & SNP2 & SNP3 \\
\hline \multirow[t]{3}{*}{$r^{2}$} & SNP1 & - & 0.825 & 0.869 & - & 0.661 & 0.728 \\
\hline & SNP2 & 0.073 & - & 0.999 & 0.049 & - & 0.999 \\
\hline & SNP3 & 0.202 & 0.399 & - & 0.097 & 0.295 & - \\
\hline
\end{tabular}

$\left|\mathrm{D}^{\prime}\right|$ above diagonal and $r^{2}$ below diagonal; $\left|\mathrm{D}^{\prime}\right|>0.5=$ shaded values. T2DM $=$ type 2 diabetes mellitus.

In the haplotype-based case-control analysis, haplotypes were established through the use of three SNPs (Table 5). The frequencies of the A-C-A haplotype were significantly higher in the type 2 diabetes patients than in the control subjects $(0.331$ vs $0.120 ; \mathrm{P}<0.001)$. The frequency of the A-C-G haplotype was markedly lower in the type 2 diabetes patients than in the control subjects $(0.160$ vs $0.365 ; \mathrm{P}<0.001)$.

Table 5. Haplotypes of the three single nucleotide polymorphisms.
\begin{tabular}{lcccccc} 
Haplotypes & T2DM (frequency) & Control (frequency) & $\chi^{2}$ & $\mathrm{P}$ & Odds ratio & $95 \%$ CI \\
\hline A-A-A & 0.355 & 0.361 & 1.132 & 0.287 & 0.944 & $0.849-1.050$ \\
A-C-A & 0.331 & 0.120 & 367.873 & $<0.001$ & 3.550 & $3.105-4.059$ \\
A-C-G & 0.160 & 0.365 & 344.758 & $<0.001$ & 0.321 & $0.284-0.363$ \\
G-A-A & - & - & - & - & - & - \\
G-C-G & 0.155 & 0.135 & 3.474 & 0.062 & 1.147 & $0.993-1.324$ \\
\hline
\end{tabular}

$\mathrm{T} 2 \mathrm{DM}=$ type 2 diabetes mellitus; $\mathrm{CI}=$ confidence interval.

\section{DISCUSSION}

The main findings in our study were in Han Chinese. T2DM patients had higher frequencies of the AA genotype of rs11558471 and the A-C-A haplotype and a lower frequency of the A-C-G haplotype than did controls.

The common forms of type 2 diabetes are thought to be complex polygenic diseases. However, it remains unknown how many genes contribute to these diseases and whether any single susceptibility gene will be shared among all ethnic groups or whether all will show some degree of population specificity. Recent high-density GWASs in humans have identified specific variants in the SLC30A8 gene that are associated with type 2 diabetes.

An understanding of the relationship between zinc and diabetes has evolved over decades. In insulin-containing vesicles of the pancreatic $\beta$ cells, crystalline insulin is packaged with zinc (MacDonald and Rorsman, 2007); in commercial preparations, insulin is crystallized with zinc to slow absorption and onset of action following subcutaneous administration. Perturbations in zinc homeostasis disrupt carbohydrate metabolism; however, the inverse relationship is also true: diabetes and hyperglycemia alter zinc balance, promoting hypozincemia and hyperzincuria (Chausmer, 1998).

Interest in the role of zinc in the pathogenesis of diabetes was reignited with the discovery, in five independent human GWASs, of an association between type 2 diabetes and a genetic polymorphism in the SLC30A8 gene (Sladek et al., 2007; Saxena et al., 2007; Scott et al., 2007; Steinthorsdottir et al., 2007; Zeggini et al., 2007). This gene codes for a newly described zinc transport protein, ZnT8 (Chimienti et al., 2004); the minor allele of the SNP 
(rs13266634) introduces a non-conservative substitution (i.e., Arg-to-Trp) in amino acid 325. This polymorphism was subsequently shown to be associated with the presence of altered glucose homeostasis, pancreatic $\beta$-cell dysfunction or overt type 2 diabetes in many study populations (Staiger et al., 2007; Horikoshi et al., 2007; Omori et al., 2008; Cauchi et al., 2008; Kirchhoff et al., 2008). So the SLC30A8 gene is thought to be a candidate gene for T2DM. In the present study, we genotyped three SNPs in the SLC30A8 gene in Han Chinese subjects, and assessed the association between SLC30A8 and T2DM using a haplotype-based case-control analysis. The AA genotype of rs11558471 significantly differed between T2DM patients and control subjects, indicating that the risk of T2DM is increased in subjects with the A allele of rs 11558471 . However, the rs11558471 is in strong linkage disequilibrium with rs13266634 (Table 1), which is associated with the increased risk of T2DM reported in several studies (Staiger et al., 2007; Horikoshi et al., 2007; Omori et al., 2008; Cauchi et al., 2008; Kirchhoff et al., 2008).

Morris and Kaplan (2002) found that for genes with multiple susceptibilities, analysis based on haplotypes has advantages over analysis based on individual SNPs. Consequently, in the present study, we successfully established haplotypes for the SLC30A8 gene from the different combinations of the three SNPs. The A-C-A haplotype was significantly higher for T2DM patients than for control subjects. However, the frequency of the A-C-G haplotype was markedly lower in type 2 diabetes patients than in control subjects.

\section{CONCLUSION}

The present results indicate that type 2 diabetes is associated with the AA genotype of rs11558471 in the human SLC30A8 gene. The A-C-A haplotype appears to be risk factor and the A-C-G haplotype may be a protective factor of type 2 diabetes in Han Chinese people.

\section{REFERENCES}

Boutayeb A and Boutayeb S (2005). The burden of non communicable diseases in developing countries. Int. J. Equity Health 4: 2.

Cauchi S, Proenca C, Choquet H, Gaget S, et al. (2008). Analysis of novel risk loci for type 2 diabetes in a general French population: the D.E.S.I.R. study. J. Mol. Med. 86: 341-348.

Chausmer AB (1998). Zinc, insulin and diabetes. J. Am. Coll. Nutr. 17: 109-115.

Chimienti F, Devergnas S, Favier A and Seve M (2004). Identification and cloning of a beta-cell-specific zinc transporter, ZnT-8, localized into insulin secretory granules. Diabetes 53: 2330-2337.

Crawford DC and Nickerson DA (2005). Definition and clinical importance of haplotypes. Annu. Rev. Med. 56: 303-320.

Das SK and Elbein SC (2006). The genetic basis of type 2 diabetes. Cell Sci. 2: 100-131.

Frayling TM (2007a). Genome-wide association studies provide new insights into type 2 diabetes aetiology. Nat. Rev. Genet. 8: 657-662.

Frayling TM (2007b). A new era in finding type 2 diabetes genes - the unusual suspects. Diabet. Med. 24: 696-701.

Horikoshi M, Hara K, Ito C, Shojima N, et al. (2007). Variations in the HHEX gene are associated with increased risk of type 2 diabetes in the Japanese population. Diabetologia 50: 2461-2466.

Kirchhoff K, Machicao F, Haupt A, Schafer SA, et al. (2008). Polymorphisms in the TCF7L2, CDKAL1 and SLC30A8 genes are associated with impaired proinsulin conversion. Diabetologia 51: 597-601.

Li Z, Zhang Z, He Z, Tang W, et al. (2009). A partition-ligation-combination-subdivision EM algorithm for haplotype inference with multiallelic markers: update of the SHEsis (http://analysis.bio-x.cn). Cell Res. 19: 519-523.

Livak KJ, Marmaro J and Todd JA (1995). Towards fully automated genome-wide polymorphism screening. Nat. Genet. 9: 341-342.

MacDonald PE and Rorsman P (2007). The ins and outs of secretion from pancreatic beta-cells: control of single-vesicle

Genetics and Molecular Research 11 (2): 1592-1598 (2012)

CFUNPEC-RP www.funpecrp.com.br 
exo- and endocytosis. Physiology 22: 113-121.

Morris RW and Kaplan NL (2002). On the advantage of haplotype analysis in the presence of multiple disease susceptibility alleles. Genet. Epidemiol. 23: 221-233.

Omori S, Tanaka Y, Takahashi A, Hirose H, et al. (2008). Association of CDKAL1, IGF2BP2, CDKN2A/B, HHEX, SLC30A8, and KCNJ11 with susceptibility to type 2 diabetes in a Japanese population. Diabetes 57: 791-795.

Owen KR and McCarthy MI (2007). Genetics of type 2 diabetes. Curr. Opin. Genet. Dev. 17: 239-244.

Palmiter RD and Huang L (2004). Efflux and compartmentalization of zinc by members of the SLC30 family of solute carriers. Pflugers Arch. 447: 744-751.

Sano M, Kuroi N, Nakayama T, Sato N, et al. (2005). Association study of calcitonin-receptor-like receptor gene in essential hypertension. Am. J. Hypertens. 18: 403-408.

Saxena R, Voight BF, Lyssenko V, Burtt NP, et al. (2007). Genome-wide association analysis identifies loci for type 2 diabetes and triglyceride levels. Science 316: 1331-1336.

Scott LJ, Mohlke KL, Bonnycastle LL, Willer CJ, et al. (2007). A genome-wide association study of type 2 diabetes in Finns detects multiple susceptibility variants. Science 316: 1341-1345.

Shi YY and He L (2005). SHEsis, a powerful software platform for analyses of linkage disequilibrium, haplotype construction, and genetic association at polymorphism loci. Cell Res. 15: 97-98.

Sladek R, Rocheleau G, Rung J, Dina C, et al. (2007). A genome-wide association study identifies novel risk loci for type 2 diabetes. Nature 445: 881-885.

Staiger H, Machicao F, Stefan N, Tschritter O, et al. (2007). Polymorphisms within novel risk loci for type 2 diabetes determine beta-cell function. PLoS One 2: e832.

Steinthorsdottir V, Thorleifsson G, Reynisdottir I, Benediktsson R, et al. (2007). A variant in CDKAL1 influences insulin response and risk of type 2 diabetes. Nat. Genet. 39: 770-775.

Xiang X, Ma YT, Fu ZY, Yang YN, et al. (2009). Haplotype analysis of the CYP8A1 gene associated with myocardial infarction. Clin. Appl. Thromb. Hemost. 15: 574-580.

Zeggini E (2007). A new era for type 2 diabetes genetics. Diabet. Med. 24: 1181-1186.

Zeggini E, Weedon MN, Lindgren CM, Frayling TM, et al. (2007). Replication of genome-wide association signals in UK samples reveals risk loci for type 2 diabetes. Science 316: 1336-1341. 